\title{
Observation of momentum-space chiral edge currents in room-temperature atoms
}

\author{
Han Cai, ${ }^{1}$ Jinhong Liu, ${ }^{1,2}$ Jinze Wu, ${ }^{1,2}$ Yanyan He, ${ }^{1}$ Shi-Yao Zhu, ${ }^{1,3}$ Jun-Xiang Zhang,,$*$ and Da-Wei Wang ${ }^{1,4, \dagger}$ \\ ${ }^{1}$ Interdisciplinary Center of Quantum Information and Department of Physics, \\ Zhejiang University, Hangzhou 310027, People's Republic of China \\ ${ }^{2}$ State Key Laboratory of Quantum Optics and Quantum Optics Devices, \\ Institute of Opto-Electronics, Shanxi University, Taiyuan 030006, People's Republic of China \\ ${ }^{3}$ Synergetic Innovation Center of Quantum Information and Quantum Physics, \\ University of Science and Technology of China, Hefei, Anhui 230026, People's Republic of China \\ ${ }^{4} \mathrm{CAS}$ Center of Excellence in Topological Quantum Computation, \\ University of Chinese Academy of Sciences, Beijing 100190, People's Republic of China
}

(Dated: August 7, 2018)

\begin{abstract}
Chiral edge currents play an important role in characterizing topological matter. In atoms, they have been observed at such a low temperature that the atomic motion can be measured. Here we report the first experimental observation of chiral edge currents in atoms at room temperature. Staggered magnetic fluxes are induced by the spatial phase difference between two standing-wave light fields, which couple atoms to form a momentum-space zigzag superradiance lattice. The chiral edge currents have been measured by comparing the directional superradiant emissions of two timed Dicke states in the lattice. This work paves the way for quantum simulation of topological matter with hot atoms and facilitates the application of topological physics in real devices.
\end{abstract}

The quantum Hall effect [1] reveals a topological class of matter that are charaterized by the Chern numbers of energy bands 2. The chiral edge currents located at the boundaries of two bulk materials with different Chern numbers are usually measured to investigate the band topology. The chirality of the edge currents is featured by the locking between the direction of the currents and the (pseudo-)spin states of the edge excitations [3, 4. The chirality is robust against local perturbations and only changes when the energy bands go through a topological transition. Since the edges have a lower dimension than the bulk, the edge currents provide a convenient platform to investigate topological physics in a higher dimension, such as the quantum Hall effect in four dimensions [5, 6]. The chirality of the edge currents persists even when a two-dimensional lattice is reduced to quasi onedimensional ribbons [7, which has been experimentally demonstrated for ultracold fermions [8, 9] and bosons [10, 11]. In those experiments, the chiral edge currents were measured with the atomic motions and thus were only observed at a low temperature, where the thermal motions are negligible.

Here we report the experimental observation of the momentum-space chiral edge currents in a superradiance lattice $12 \sqrt{14}$ of cesium atoms at room temperature. The zigzag lattice that we have synthesized is similar to the ladder structures in the experiments with cold atoms [7, 8, 11] and is currently under intensive investigation [15 17. Different from the momentum-space lattices characterized by the recoil momentum of cold atoms [17, 18, the superradiance lattice is a momentum-space lattice composed by the timed Dicke states [19, which are collective atomic excitations with phase correlations. The phase correlations can be understood as the momenta of the collective excitations, which have directional
A

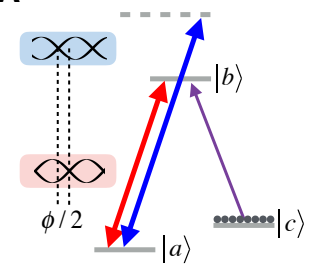

B

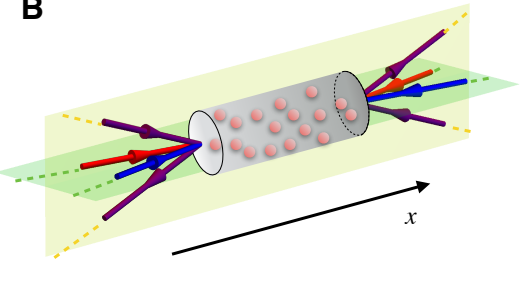

Figure 1: Schematic configuration of the experiment. (A) Atomic levels and the near-resonant and far-detuned standing waves coupling fields with a relative spatial phase difference $\phi / 2$. (B) The configuration of the lasers. Both the far-detuned (blue) and near-resonant (red) standing wave coupling fields have an angle to the $\hat{x}$-axis in order to satisfy the phase-matching condition. The plane formed by the two standing wave coupling light beams is perpendicular to the one formed by the incident and reflected probe beams.

superradiant light emissions when they satisfy the phasematching condition with a light mode [19. A remarkable advantage of our approach is that the edge currents are observed at room temperature. Instead of measuring the atomic motions [8 11], the chiral edge currents are measured by comparing the directional light emissions from two timed Dicke states. Strikingly, the chiral edge currents are robust to room-temperature thermal motions, which only induce an average effect of the chiral edge currents in the real-space Brillouin zone. Our study has substantially lowered the threshold of the experimental observation of chiral edge currents in atoms.

To highlight the physics, we introduce our basic model with $\Lambda$-type three-level atoms as shown in Fig. 1 (A). An excited state $|b\rangle$ and a metastable state $|a\rangle$ are coupled by two standing waves with different frequencies, i.e., a nearresonant and a far-detuned standing waves with field am- 
plitude envelopes $\cos \left(k_{c} x\right)$ and $\cos \left(k_{c} x+\phi / 2\right)$, where $\phi / 2$ is their spatial phase difference. The detuning between the two standing wave coupling fields is small enough such that the difference between the amplitudes of their wavevectors $k_{c}$ can be neglected. A probe field couples the ground state $|c\rangle$ to $|b\rangle$. The Hamiltonian is (we set $\hbar=1)$,

$$
\begin{aligned}
H= & \sum_{m}\left[\frac{\Delta_{c}}{2}+2 \kappa \cos \left(2 k_{c} x+\phi\right)\right]\left(\left|a_{m}\right\rangle\left\langle a_{m}|-| b_{m}\right\rangle\left\langle b_{m}\right|\right) \\
& +\sum_{m} 2 \Omega \cos \left(k_{c} x_{m}\right)\left(\left|b_{m}\right\rangle\left\langle a_{m}\right|+h . c .\right) \\
& +\sum_{m}\left[\Omega_{p} e^{i k_{p} x_{m}} e^{-i\left[\Delta_{p}-\left(\nu_{c}-\omega_{b a}\right) / 2\right] t}\left|b_{m}\right\rangle\left\langle c_{m}\right|+h . c .\right],
\end{aligned}
$$

where $\Delta_{c}=\nu_{c}-\omega_{b a}+4 \kappa$ is the detuning of the nearresonant coupling field frequency $\nu_{c}$ from the atomic transition between $|b\rangle$ and $|a\rangle . \omega_{b a}$ is the bare transition frequency and $4 \kappa$ is a spatially homogeneous Stark shift induced by the far-detuned coupling fields, which also induce a spatially periodic Stark shift $2 \kappa \cos \left(2 k_{c} x+\phi\right)$. Here $\kappa=\Omega_{f}^{2} / \Delta_{f}$ with $\Omega_{f}$ and $\Delta_{f}=\nu_{f}-\omega_{b a}$ being the Rabi frequency and detuning of each plane wave component of the far-detuned coupling field. $\Delta_{p}=\nu_{p}-\omega_{b c}$ is the detuning of the probe field frequency $\nu_{p}$ from the atomic transition frequency $\omega_{b c}$ between $|b\rangle$ and $|c\rangle . \Omega$ is the Rabi frequency of each plane wave component of the near-resonant coupling fields. $k_{p(c)}$ is the $\hat{x}$-component of the probe (coupling) field wavevector and $x_{m}$ is the $\hat{x}$-axis coordinate of the $m^{\text {th }}$ atom. The derivation of the Hamiltonian in Eq. (1) can be found in Sec. I of the Supplementary Material.

By introducing collective atomic excitation operators $\hat{b}_{j}^{\dagger}=1 / \sqrt{N} \sum_{m} e^{i\left(k_{p}+2 j k_{c}\right) x_{m}}\left|b_{m}\right\rangle\left\langle c_{m}\right|$ and $\hat{a}_{j}^{\dagger}=$ $1 / \sqrt{N} \sum_{m} e^{i\left[k_{p}+(2 j-1) k_{c}\right] x_{m}}\left|a_{m}\right\rangle\left\langle c_{m}\right|$, we transform the Hamiltonian to momentum space, $H=H_{s}+H_{p}$ where

$$
\begin{aligned}
H_{s}= & \sum_{j} \Delta_{c}\left(\hat{a}_{j}^{\dagger} \hat{a}_{j}-\hat{b}_{j}^{\dagger} \hat{b}_{j}\right) / 2+\sum_{j}\left[\Omega\left(\hat{a}_{j}^{\dagger} \hat{b}_{j}+\hat{a}_{j}^{\dagger} \hat{b}_{j-1}\right)\right. \\
& \left.+e^{i \phi} \kappa\left(\hat{a}_{j}^{\dagger} \hat{a}_{j-1}-\hat{b}_{j}^{\dagger} \hat{b}_{j-1}\right)+h . c .\right],
\end{aligned}
$$

and $H_{p}=\sqrt{N} \Omega_{p}\left[\hat{b}_{0}^{\dagger} e^{-i\left[\Delta_{p}-\left(\nu_{c}-\omega_{b a}\right) / 2\right] t}+\right.$ h.c. $]$. With the condition that $\Omega \gg \Omega_{p}$, most of the atoms are in the ground state $|c\rangle$, and $\hat{a}_{j}^{\dagger}$ and $\hat{b}_{j}^{\dagger}$ are bosonic [12, 20]. For single excitations, Eq. (2) is a Hamiltonian of a tight-binding superradiance lattice composed by timed Dicke states, $\left|B_{j}\right\rangle \equiv \hat{b}_{j}^{\dagger}\left|c_{1}, c_{2}, \ldots, c_{N}\right\rangle$ and $\left|A_{j}\right\rangle \equiv$ $\hat{a}_{j}^{\dagger}\left|c_{1}, c_{2}, \ldots, c_{N}\right\rangle$. For more excitations, as long as the excitation number is much less than the atomic number, the physics remains the same due to the bosonic nature of the excitations.

The phase factor $e^{i \phi}$ in Eq. (2) is induced by the spatial phase difference between the two standing wave coupling fields. Each up and down triangle encloses an effective magnetic flux $\phi$ and $\pi-\phi$, respectively, as shown
A

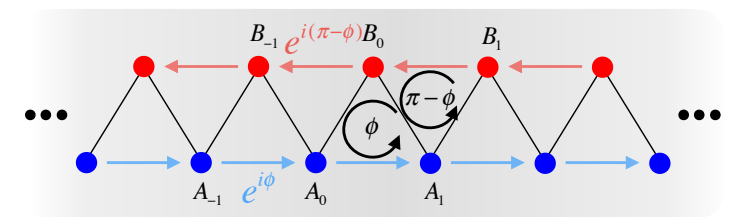

B

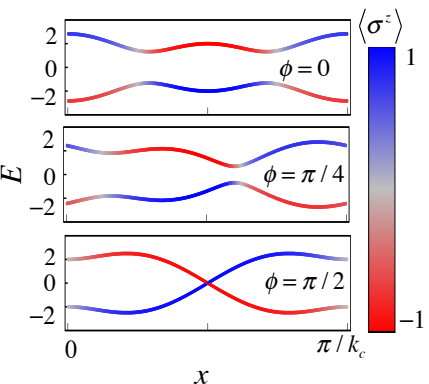

D

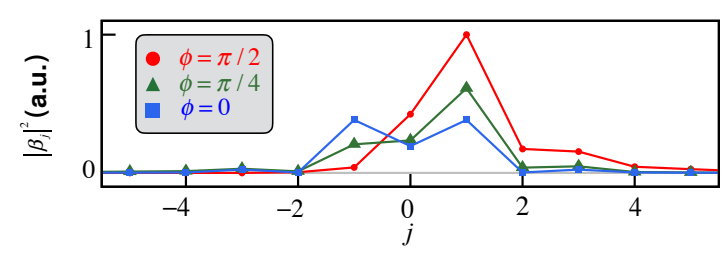

Figure 2: The superradiance lattice and its band structure with different magnetic fluxes. (A) The tightbinding lattice of the Hamiltonian $H_{s}$ in Eq. (2). The red and blue arrows show the phase factors $\phi$ attached with the corresponding transitions. The total phases enclosed by the loop transitions in the down and up triangles are $\phi$ and $\pi-\phi$, respectively. The relative phase $\phi=\pi / 2$. (B) The dispersion relation according to Eq. (3) with $\phi=0, \pi / 4$ and $\pi / 2$. The colour shows the $\left\langle\sigma_{z}\right\rangle$ of eigenstates and indicates which edge the eigenstates are mainly located on. (C) $J_{b}$ in Eq. (4) for $\phi=0$ (blue square), $\pi / 4$ (green triangle) and $\pi / 2$ (red circle). (D) The steady state distribution of the population in the $|b\rangle$-sublattice with a probe field pumping the atoms from the ground state to the state $\left|B_{0}\right\rangle$. The decoherence rates of states $|b\rangle$ and $|a\rangle$ are $\gamma_{b c}=1$ and $\gamma_{a c}=0.1$. The probe detuning $\Delta_{p}=0 . \Omega=1, \kappa=1$ and $\Delta_{c}=0$.

in Fig. 2 (A). A direct consequence of this effective magnetic field is the chiral edge currents, which can be demonstrated by the dispersion relation of $H_{s}$. We diagonalize $H_{s}$ in real space,

$$
H_{s}=h \mathbf{n} \cdot \sigma,
$$

where $\mathbf{n}=\left(h_{x} \hat{x}+h_{z} \hat{z}\right) / h$ with $h_{x}=2 \Omega \cos \left(k_{c} x\right)$, $h_{z}=\Delta_{c} / 2+2 \kappa \cos \left(2 k_{c} x+\phi\right)$, and $h=\sqrt{h_{z}^{2}+h_{x}^{2}}$. $\sigma=\sum_{j=x, y, z} \sigma^{j} \hat{j}$ is the vector of the Pauli matrices of the pseudo spin-up state $|a\rangle$ and spin-down state $|b\rangle$. The dispersion relations in the two bands are $E_{ \pm}= \pm h$ with the eigenstates $\left|\psi_{+}\right\rangle=\cos (\theta / 2)|a\rangle+\sin (\theta / 2)|b\rangle$ and $\left|\psi_{-}\right\rangle=-\sin (\theta / 2)|a\rangle+\cos (\theta / 2)|b\rangle$, where $\theta$ is the polar angle of $\mathbf{n}$. In Fig. $2(\mathbf{B})$, we plot the dispersion with the "spin texture" $\left\langle\sigma^{z}\right\rangle$. For $\phi=\pi / 2$, most eigenstates concentrate on one of the two edges.

The evolution of the momentum is determined by the dispersion relation in Fig. $2(\mathbf{B}), \partial p_{ \pm} / \partial t=-\partial E_{ \pm} / \partial x$ 

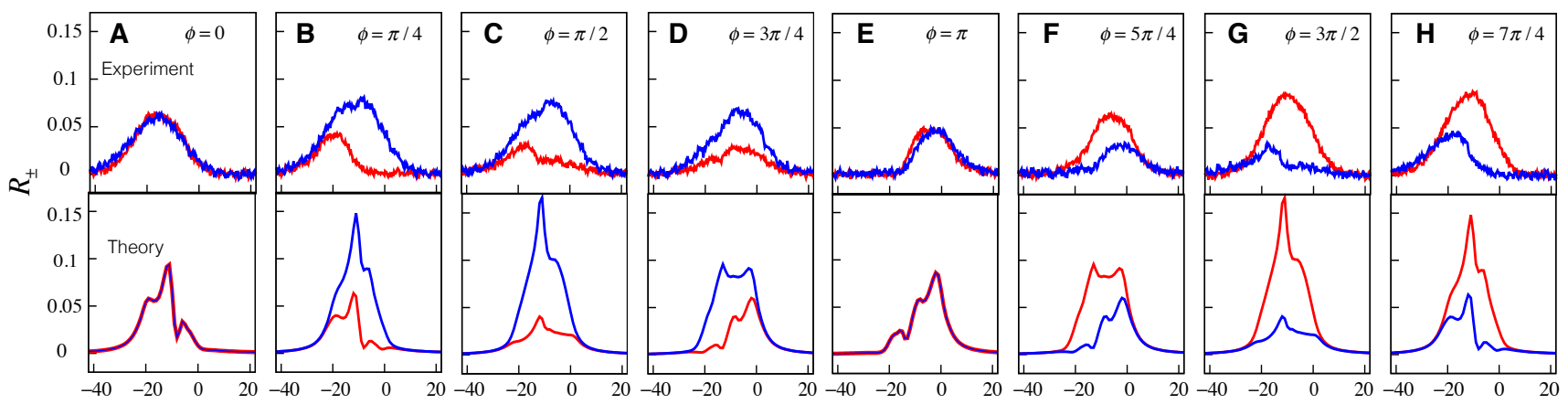

$\Delta_{p}(\mathrm{MHz})$

Figure 3: The chiral edge currents demonstrated by the reflection spectra. $R_{+}$are red lines and $R_{-}$are blue lines with phases $(\mathbf{A}) \phi=0,(\mathbf{B}) \phi=\pi / 4,(\mathbf{C}) \phi=\pi / 2,(\mathbf{D}) \phi=3 \pi / 4,(\mathbf{E}) \phi=\pi,(\mathbf{F}) \phi=5 \pi / 4,(\mathbf{G}) \phi=3 \pi / 2$ and $(\mathbf{H}) \phi=7 \pi / 4$. The power of each plane wave component of the near-resonant coupling field is $6.5 \mathrm{~mW}$ with an effective Rabi frequency $\Omega=5.5$ MHz. The power of each plane wave component of the far-detuned coupling field is $120 \mathrm{~mW}$ with an effective Rabi frequency $\Omega_{f}=33.3 \mathrm{MHz}$ and a detuning $\Delta_{f}=200 \mathrm{MHz} . \kappa=5.5 \mathrm{MHz} . \Delta_{c}=1 \mathrm{MHz}$. The power of each probe beam is $20 \mu \mathrm{W}$.

12. For example, when $\phi=\pi / 2$ and near the energy $E=0$, the momentum of an excitation created on the $|b\rangle$ sublattice increases with time (note the negative derivative of the red line), while on $|a\rangle$-sublattices the momentum decreases. This is the essence of chiral edge currents, i.e., excitations on different edges or with different spin states move in opposite directions. To quantitatively clarify this feature, we define the chiral edge currents on the $|b\rangle$-sublattice as [7,

$$
J_{b}(E)=-\sum_{i= \pm} \int \mathrm{d} x \delta\left(E_{i}-E\right)\left|\left\langle\psi_{i} \mid b\right\rangle\right|^{2} \frac{\partial E_{i}}{\partial x}
$$

where $\delta\left(E_{i}-E\right)$ is the Dirac delta function. $J_{b}(E)$ characterizes the dynamics of the total momentum of excitations with energy $E$ on the $|b\rangle$-sublattice. We can define a similar quantity for the $|a\rangle$-sublattice. However, we only focus on $J_{b}(E)$ since the probe field couples atoms to the $|b\rangle$ state. In Fig. 2 $(\mathbf{C})$, we plot $J_{b}(E)$ for different phases $\phi$. For $\phi=0, J_{b}(E)=0$. For $\phi=\pi / 4, J_{b}(E)$ is positive in the two bands. $J_{b}(E)$ is almost a big positive constant when $\phi=\pi / 2$. When $\phi=\pi, J_{b}(E)=0$ and when $\phi=3 \pi / 2, J_{b}(E)$ is negative to the one with $\phi=\pi / 2$ (see Sec. II of the Supplementary Material).

To generate $J_{b}(E)$, we apply a weak probe field with detuning $\Delta_{p}=E+\left(\nu_{c}-\omega_{b a}\right) / 2$ to pump the atoms from the ground state to the state $\left|B_{0}\right\rangle$. The excitation induces $J_{b}(E)$ and is finally balanced by the decoherence of the atomic states. In the steady state, the population distribution is plotted in Fig. 2 (D). We define $\beta_{j}$ as the probability amplitude of the state $\left|B_{j}\right\rangle$. For $\phi=0$, the distribution of $\left|\beta_{j}\right|^{2}$ is symmetric on both sides of $\left|B_{0}\right\rangle$, in contrasted to the asymmetric distribution when $\phi=\pi / 4$ and $\phi=\pi / 2$, where the population is biased to $j>0$. The result is consistent with Fig. 2 (C).

In the experiment, we detect the superradiant emissions of two specific timed Dicke states to show the edge currents. The timed Dicke states with a phase correlation that matches the wavevectors of the light in the medium, i.e., $\left|k_{p}+2 j k_{c}\right| \approx\left|k_{p}\right|$, have directional superradiant emissions [19. In the current scheme where $|a\rangle$ and $|c\rangle$ are nearly degenerate, the timed Dicke state $\left|B_{-1}\right\rangle$ (or $\left|B_{+1}\right\rangle$ ) is the only superradiant state besides $\left|B_{0}\right\rangle$ for a probe light incident along $+\hat{x}$ (or $-\hat{x}$ ). The radiation from these two superradiant states can be considered as the reflectivities $R_{ \pm}$of the probe fields incident to the atoms along $\pm \hat{x}$. The relationship between $R_{ \pm}$and $\beta_{ \pm 1}$ is (see Methods and a rigorous calculation in Sec. III of the Supplementary Material),

$$
\frac{R_{+}}{R_{-}}=\frac{\left|\beta_{-1}\right|^{2}}{\left|\beta_{+1}\right|^{2}} .
$$

This relation is independent of the density of the atoms, the length of the vapor cell and the phase mismatch.

We have used the D1 line of cesium atoms in the experiment: $|a\rangle=\left|6^{2} S_{1 / 2}, F=3\right\rangle,|b\rangle=\left|6^{2} P_{1 / 2}, F=4\right\rangle$ and $|c\rangle=\left|6^{2} S_{1 / 2}, F=4\right\rangle$ (see Methods for details of the experiment). Typical experimental results are shown in Fig. 3. The reflection spectra depend on the phase $\phi$. Only when $\phi=0$ and $\pi$, we observe $R_{+}=R_{-}$, as shown in Figs. 3 (A) and (E). For $0<\phi<\pi$, we observe $R_{+}<R_{-}$, as shown in Figs. $3(\mathbf{B})-(\mathbf{D})$, which indicates a larger population in $\left|B_{+1}\right\rangle$ than in $\left|B_{-1}\right\rangle$, resulted from an edge current propagating along $+\hat{x}$. This is consistent with the results in Figs. $2(\mathbf{C})$ and $(\mathbf{D})$. In contrast, we observe $R_{+}>R_{-}$for $\pi<\phi<2 \pi$, as shown in Figs. 3 $(\mathbf{F})-(\mathbf{H})$, which indicates an edge current along $-\hat{x}$. The differences between the simulation and experiment are attributed to slight asymmetry in the optical alignment, an average of $\phi$ along the vapour cell $(\Delta \phi \approx 0.05 \pi)$ and the Gaussian rather than plane wave profiles of the coupling fields.

In the following, we analyse the robustness of the edge 

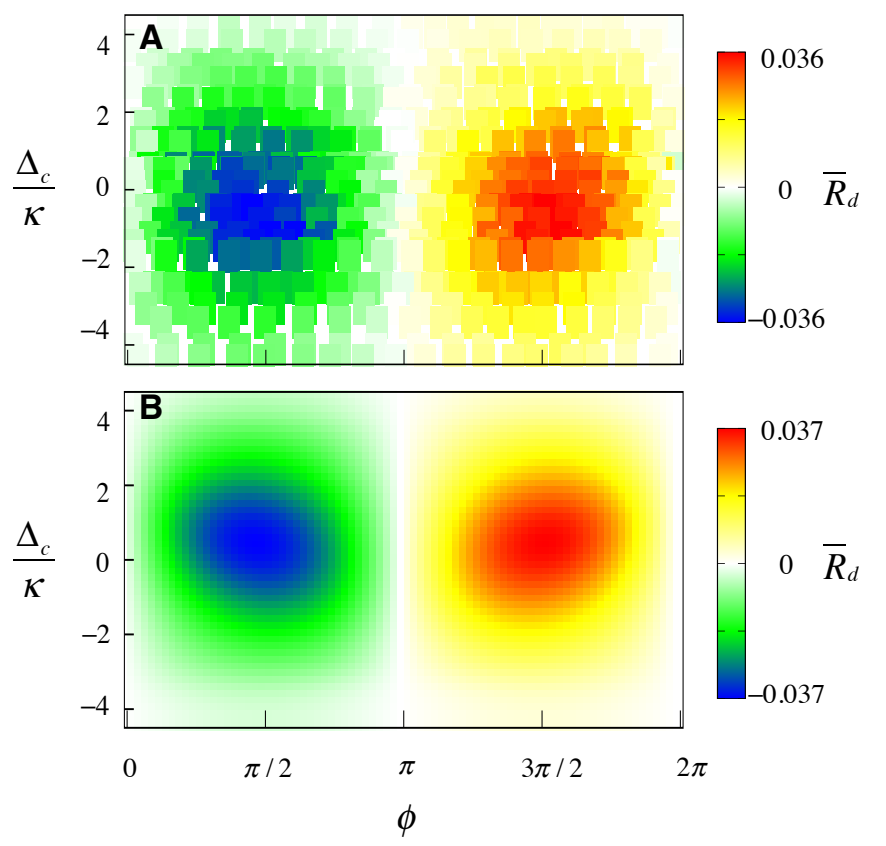

Figure 4: The averaged difference between the two reflectivities in Eq. (6). (A) Experimental data. (B) Numerical simulation. The parameters are the same as in Fig. 3

currents against thermal motions. From Fig. 3, we see that the reflection spectra are not Doppler broadened. The non-zero region of the spectra coincides with the energy bands of the superradiance lattice. Their scales are both around $30 \mathrm{MHz}$. This feature of standing-wave coupled electromagnetically induced transparency was already found by Feldman and Feld in 1972 21. To understand this, we notice that the atoms that have a Doppler shift larger than the bandwidth is out of resonance with the probe field no matter their positions such that they cannot be excited. For atoms with Doppler shift smaller than the lattice bandwidth, they move in the real-space Brillouin zone and their contribution to the edge currents needs to be averaged with their positions. The chiral edge currents induce a difference between the populations on $\left|B_{-1}\right\rangle$ and $\left|B_{+1}\right\rangle$, and subsequently a difference between the two reflectivities $R_{d} \equiv R_{+}-R_{-} \propto\left|\left\langle\beta_{-1}\right\rangle\right|^{2}-\left|\left\langle\beta_{+1}\right\rangle\right|^{2}$ where \langle\rangle is the average over the Doppler shifts due to the thermal motions of the atoms. To quantify the effect of the chiral edge currents on the whole reflection spectra, we make average of $R_{d}$,

$$
\bar{R}_{d}=\frac{1}{F} \int R_{d} \mathrm{~d} \Delta_{p}
$$

where $F=40 \mathrm{MHz}$ is the frequency range of an integration from $-30 \mathrm{MHz}$ to $10 \mathrm{MHz}$ in the reflection spectra. In Fig. 4, we show the experimental data and numerical simulation of $\bar{R}_{d}$ as functions of $\Delta_{c}$ and $\phi$. For an on-site potential difference $\Delta_{c} \approx 0, \bar{R}_{d}$ is approximately a sinusoidal function of the phase $\phi$. This means our method can be used to measure a phase difference between two standing waves. For $\Delta_{c}$ larger than the bandwidth, the inter-edge transitions are inhibited and the effect of the synthetic magnetic field diminishes due to the inefficient loop transitions. As a result, $\bar{R}_{d}$ decreases rapidly to zero when $\Delta_{c}$ increases. The results in Fig. 4 can be understood as the phase diagram of an extended Haldane model [7] and its relation to the dynamic classification of topological phases [22] will be discussed elsewhere.

The results reported here are substantially different from the temperature-independent edge currents in the photonic lattices 23 27, where the propagation of the photons governed by the Maxwell equations is made analogy to the Schrödinger equations [28. The edge states there are photonic states rather than atomic states. The temperature has no influence on the photons. In our current study, the topological bands are for the atoms and they intrinsically obey the Schrödinger equation. The thermal motions of the atoms make a convenient average of the edge currents. In addition, our lattice is in momentum space, in contrast to the real-space topological photonic lattices. Although the edge currents are detected by light in our experiment, they are currents of collective excitations of atoms in momentum space, not light in real space.

On the other hand, our results are closely related to the spin-orbit coupled system [29, 30] and the momentumspace lattice [17, 18] in cold atoms, with the difference that the momentum is represented by the phase correlation of the collective excitation, instead of the recoil momentum, which is negligible in our study. An extension of our model to higher dimensions [13] can be used to simulate the Haldane model [31, 32] and the two-dimensional spin-orbit coupling [29, 30]. By using Rydberg states [33, 34] we can introduce interactions between the excitations and study the many-body effect in flux lattices [35]. An interesting connection of our results can also be made to the unidirectional reflectionless (invisible) photonic structures [36 39], such as the paritytime symmetric materials [40]. We have observed that under certain conditions one of the two reflectivities is nearly zero while the other is big. Another observation is that the transmission of the probe fields in the two opposite directions are the same, although the transmissions are phase-dependent. This is because our system does not break the time-reversal symmetry. It is interesting to note that an effective magnetic field in momentum space does not result in optical nonreciprocity, while an effective electric field in momentum space can break the time-reversal symmetry and induce optical nonreciprocity [1]. Our study provides a new way to measure the spatial relative phase between two light fields that have different frequencies. The phase information is converted to intensity signals. This can be used in phase-contrast microscopy.

We thank B. Gadway, J. Q. You and G. Juzeliunas 
for fruitful discussion. We acknowledge the support from the National Key Research and Development Program of China under Grant No.2018YFA0307200, the Joint Fund of National Natural Science Foundation of China (U1330203) and National Natural Science Foundation of China (No.91736209, 11574188).

* Electronic address: junxiangzhang@zju.edu.cn

$\dagger$ Electronic address: dwwang@zju.edu.cn

[1] Klitzing, K. v., Dorda, G., and Pepper, M. Physical Review Letters 45(6), 494-497 (1980).

[2] Thouless, D. J., Kohmoto, M., Nightingale, M. P., and Dennijs, M. Physical Review Letters 49(6), 405-408 (1982).

[3] Kane, C. L. and Mele, E. J. Physical Review Letters 95(22), 226801 (2005).

[4] Bernevig, B. A. and Zhang, S.-C. Physical Review Letters 96(10), 106802 (2006).

[5] Lohse, M., Schweizer, C., Price, H. M., Zilberberg, O., and Bloch, I. Nature 553, 55 (2018).

[6] Zilberberg, O., Huang, S., Guglielmon, J., Wang, M., Chen, K. P., Kraus, Y. E., and Rechtsman, M. C. Nature 553, 59 (2018).

[7] Hügel, D. and Paredes, B. Physical Review A 89(2), 023619 (2014).

[8] Mancini, M., Pagano, G., Cappellini, G., Livi, L., Rider, M., Catani, J., Sias, C., Zoller, P., Inguscio, M., Dalmonte, M., and Fallani, L. Science 349(6255), 1510-1513 (2015).

[9] Livi, L. F., Cappellini, G., Diem, M., Franchi, L., Clivati, C., Frittelli, M., Levi, F., Calonico, D., Catani, J., Inguscio, M., and Fallani, L. Physical Review Letters 117(22), 220401 (2016).

[10] Atala, M., Aidelsburger, M., Lohse, M., Barreiro, J. T., Paredes, B., and Bloch, I. Nature Physics 10(8), 588-593 (2014).

[11] Stuhl, B. K., Lu, H.-I., Aycock, L. M., Genkina, D., and Spielman, I. B. Science 349(6255), 1514-1518 (2015).

[12] Wang, D.-W., Liu, R.-B., Zhu, S.-Y., and Scully, M. O. Physical Review Letters 114(4), 043602 (2015).

[13] Wang, D.-W., Cai, H., Yuan, L., Zhu, S.-Y., and Liu, R.-B. Optica 2(8), 712-715 (2015).

[14] Chen, L., Wang, P., Meng, Z., Huang, L., Cai, H., Wang, D.-W., Zhu, S.-Y., and Zhang, J. Physical Review Letters 120, 193601 (2018).

[15] Anisimovas, E., Račiūnas, M., Sträter, C., Eckardt, A., Spielman, I., and Juzeliūnas, G. Physical Review A 94(6), 063632 (2016).

[16] Xu, J., Gu, Q., and Mueller, E. J. Physical Review Letters $\mathbf{1 2 0}(8), 085301$ (2018).

[17] An, F. A., Meier, E. J., Ang'ong'a, J., and Gadway, B. Physical Review Letters 120(4), 040407 (2018).

[18] An, F. A., Meier, E. J., and Gadway, B. Science Advances 3(4) (2017).

[19] Scully, M. O., Fry, E. S., Ooi, C. H. R., and Wódkiewicz, K. Physical Review Letters 96(1), 010501 (2006).

[20] Fleischhauer, M. and Lukin, M. D. Physical Review Letters 84(22), 5094-5097 (2000).

[21] Feldman, B. J. and Feld, M. S. Physical Review A 5(2), 899.
[22] Zhang, L., Zhang, L., Niu, S., and Liu, X.-J. ArXiv 1802.10061 (2018).

[23] Rechtsman, M. C., Zeuner, J. M., Plotnik, Y., Lumer, Y., Podolsky, D., Dreisow, F., Nolte, S., Segev, M., and Szameit, A. Nature 496(7444), 196-200 (2013).

[24] Hafezi, M., Mittal, S., Fan, J., Migdall, A., and Taylor, J. M. Nature Photonics 7, 1001 (2013).

[25] Zhang, Y., Wu, Z., Belić, M. R., Zheng, H., Wang, Z., Xiao, M., and Zhang, Y. Laser \& Photonics Reviews 9(3), 331-338 (2015).

[26] Xiao, L., Zhan, X., Bian, Z. H., Wang, K. K., Zhang, X., Wang, X. P., Li, J., Mochizuki, K., Kim, D., Kawakami, N., Yi, W., Obuse, H., Sanders, B. C., and Xue, P. Nature Physics 13(11), 1117-1123 (2017).

[27] Bandres, M. A., Wittek, S., Harari, G., Parto, M., Ren, J., Segev, M., Christodoulides, D. N., and Khajavikhan, M. Science. 10.1126/science.aar4005.

[28] Haldane, F. D. M. and Raghu, S. Physical Review Letters 100(1), 013904 (2008).

[29] Wu, Z., Zhang, L., Sun, W., Xu, X. T., Wang, B. Z., Ji, S. C., Deng, Y. J., Chen, S., Liu, X. J., and Pan, J. W. Science 354(6308), 83-88 (2016).

[30] Huang, L., Meng, Z., Wang, P., Peng, P., Zhang, S.-L., Chen, L., Li, D., Zhou, Q., and Zhang, J. Nature Physics 12, 540 (2016).

[31] Haldane, F. D. M. Physical Review Letters 61(18), 20152018 (1988).

[32] Jotzu, G., Messer, M., Desbuquois, R., Lebrat, M., Uehlinger, T., Greif, D., and Esslinger, T. Nature 515(7526), 237-240 (2014).

[33] Peyronel, T., Firstenberg, O., Liang, Q. Y., Hofferberth, S., Gorshkov, A. V., Pohl, T., Lukin, M. D., and Vuletic, V. Nature 488(7409), 57-60 (2012).

[34] Ripka, F., Chen, Y.-H., Löw, R., and Pfau, T. Physical Review A 93(5), 053429 (2016).

[35] Greschner, S. and Vekua, T. Physical Review Letters 119(7), 073401 (2017).

[36] Lin, Z., Ramezani, H., Eichelkraut, T., Kottos, T., Cao, H., and Christodoulides, D. N. Physical Review Letters 106(21), 213901 (2011).

[37] Regensburger, A., Bersch, C., Miri, M.-A., Onishchukov, G., Christodoulides, D. N., and Peschel, U. Nature 488, 167 (2012).

[38] Wu, J.-H., Artoni, M., and La Rocca, G. C. Physical Review Letters 113(12), 123004 (2014).

[39] Huang, Y., Shen, Y., Min, C., Fan, S., and Veronis, G. Nanophotonics 6(5), 977 (2017).

[40] Feng, L., El-Ganainy, R., and Ge, L. Nature Photonics 11(12), 752-762 (2017).

[41] Wang, D.-W., Zhou, H.-T., Guo, M.-J., Zhang, J.-X., Evers, J., and Zhu, S.-Y. Physical Review Letters 110(9), 093901 (2013).

\section{METHODS}

\section{A. Experiment}

The experimental set-up is schematically drawn in Fig. 5. A Ti:Sapphire laser operating at ${ }^{137} \mathrm{Cs} D_{1}$ line $(895$ $\mathrm{nm}$ ) is split by a polarization beam splitter (PBS) into two beams for the two coupling fields. The vertically po- 


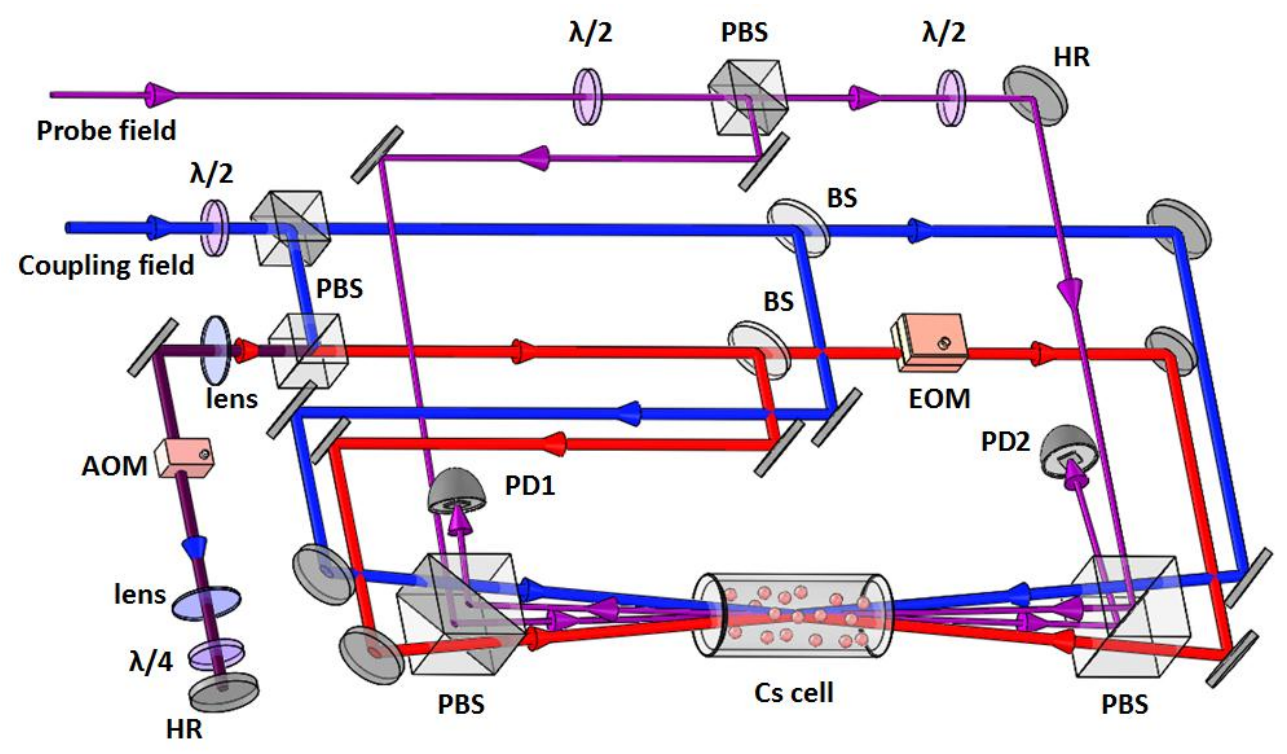

Figure 5: The experimental set-up. BS: beam splitter. PBS: polarization beam splitter. AOM: acousto-optical modulator. EOM: elctro-optic modulator. HR: high reflection mirror. PD: photo detector. $\lambda / 2$ : half-wave plate. $\lambda / 4$ : quarter-wave plate.

larized beam is detuned by an acousto-optical modulator $(\mathrm{AOM})$ in a double-pass configuration, during which its polarization is rotated to the horizontal direction by a quarter-wave plate. The two coupling fields are then split by $50 / 50$ beam splitters (BS) to form two standing waves. The laser that is detuned by the AOM serves as the near-resonant coupling field. A plane wave component of the far-detuned standing wave goes through an electro-optic modulator (EOM) to tune the spatial relative phase $\phi / 2$ between the two standing waves. Each plane wave component of the near-resonant coupling field is aligned to overlap with the counterpropagating component of the far-detuned coupling field. A Toptica DL100 semiconductor laser with vertical polarization is split by a 50/50 BS to generate two counter-propagating probe beams. The probe fields and two coupling fields form a triangular configuration along their propagation direction, as shown in Fig. 1 (B). The intersection angles between the probe field and the two coupling fields are both 0.62 degree. The intersection angle between the two coupling fields is 1.0 degree. These angles make sure the phase-matching condition can be satisfied assisted by the dispersion of the atomic gas. In addition, the nondegenerate wave-mixing signals are phase matched in a different direction from the degenerate wave-mixing signals that we detect. The $e^{-2}$ full width of the coupling field is $1.6 \mathrm{~mm}$ while the one for the probe field is 0.5 $\mathrm{mm}$. The length of the atomic vapour cell is $2 \mathrm{~cm}$. The probe beam is totally covered by the coupling beams in the atomic vapour. The cell length is small enough that we consider the relative phase between the two stand- ing waves is a constant. The two reflected signals were simultaneously recorded with photodiode detectors.

\section{B. Numerical simulation}

The reflectivities $R_{ \pm}$are obtained from the coupledwave equations [13,

$$
\begin{aligned}
& \frac{\partial E_{p}}{\partial x}=-\eta \operatorname{Im} \beta_{0} E_{p}+i \eta \beta_{+1} e^{-i \Delta k x} E_{r} \\
& \frac{\partial E_{r}}{\partial x}=+\eta \operatorname{Im} \beta_{0} E_{r}-i \eta \beta_{-1} e^{+i \Delta k x} E_{p}
\end{aligned}
$$

where $E_{p}$ and $E_{r}$ are the slowly-varying field amplitudes of the probe field propagating in $+\hat{x}$ and the reflected field. $\eta$ is a parameter relating the probability amplitudes to the susceptibilities [13. 'Im' stands for the imaginary part. $\Delta k=2\left(k_{p}-k_{c}\right)$ is the wavevector mismatch in $\hat{x}$ direction. By setting the boundary condition $E_{p}(0)=E_{0}$, $E_{r}(L)=0$ where $L$ is the length of the cell, we obtain $R_{+}=\left|E_{r}(0) / E_{0}\right|^{2}$ from the coupled wave equations. On the other hand, by setting $E_{p}(0)=0, E_{r}(L)=E_{0}$, we obtain $R_{-}=\left|E_{r}(0) / E_{0}\right|^{2}$. In the Supplementary Material, we show that the coupled-wave equation results in the simple relation in Eq. (5).

In calculating $\beta_{j}$, we have used the master equation that takes into account the decoherence and Doppler shifts of the atoms (see Supplementary Material). We adopt a more rigorous approach based on calculating the Fourier components of the atomic coherences in the reference frame of moving atoms. 